\title{
Supporting Fuzzy Rough Sets in Fuzzy Description Logics
}

\author{
Fernando Bobillo ${ }^{1}$ and Umberto Straccia ${ }^{2}$ \\ 1 Dpt. of Computer Science \& Systems Engineering, University of Zaragoza, Spain \\ ${ }^{2}$ Istituto di Scienza e Tecnologie dell'Informazione (ISTI - CNR), Pisa, Italy \\ fbobillo@unizar.es, straccia@isti.cnr.it
}

\begin{abstract}
Classical Description Logics (DLs) are not suitable to represent vague pieces of information. The attempts to achieve a solution have lead to the birth of fuzzy DLs and rough DLs. In this work, we provide a simple solution to join these two formalisms and define a fuzzy rough DL. We also show how to extend two reasoning algorithms for fuzzy DLs, which are implemented in the fuzzy DL reasoners FUZzYDL and DeLOREAN.
\end{abstract}

\section{Introduction}

In the last years the interest in ontologies has significantly grown. An ontology is defined as an explicit and formal specification of a shared conceptualization [13], which means that ontologies represent the vocabulary of some domain. They have gained widespread popularity due to their success in several applications such as expert and multiagent systems or the Semantic Web. Description Logics (DLs) are a family of logics for representing structured knowledge [1. They are the basis of most of the ontology languages, such as the current standard language OWL [16]. For instance, the logic behind the recent language OWL 2 is $\mathcal{S R O I \mathcal { Q }}(\mathbf{D})$ [8.

However, it is widely agreed that "classical" ontology languages are not appropriate to deal with fuzzy/vague/imprecise knowledge, which is inherent to several real world domains. With the aim of managing vagueness in ontologies, several extension of DLs have been proposed, being possible to group them in two categories. On the one hand, the combination with fuzzy logic [30] produced fuzzy DLs. Some notable works are [15 25 2628], for a survey we refer to 21. Under this approach, vagueness is quantified and expressed using a degree of membership to a vague concept. On the other hand, the combination with rough set theory [22] produced rough DLs [10]12]1819|20|24]. These logics offer a qualitative approach to model vagueness. Instead of providing a degree of a membership, vague concepts are approximated by means of a couple of classical sets: an upper and a lower approximation. This approach is very useful when it is not possible to quantify the membership function of a vague concept.

Fuzzy logic and rough logic are complementary formalism to manage vagueness and hence it is natural to combine them by means of fuzzy rough sets [1123.

C. Sossai and G. Chemello (Eds.): ECSQARU 2009, LNAI 5590, pp. 676 687, 2009.

(C) Springer-Verlag Berlin Heidelberg 2009 
This is useful in several domains of application. For instance, in e-commerce, it is possible to combine rough concepts such as "potential buyer" (an individual which is possibly interested in some product) with fuzzy concepts such as "cheap price" (which can be modeled with a trapezoidal membership function). Another example is medicine, which combines rough concepts such as "possible patient" (an individual affected by some of the symptoms of some disease, and hence suspected of being patient) with fuzzy concepts such as "high blood pressure".

In this paper we follow this approach and extend a fuzzy DL with fuzzy rough sets. As we will see, the integration is seamless, as already pointed out by [24] for the classical semantics case, as the rough set component can mapped into the fuzzy DL component, with the non-negligible advantage that current fuzzy DLs reasoners can be used with minimal adaption.

Related works are 9, which presents a rough fuzzy ontology but without entering into the formal details of the subjacent logic, and [17], which considers a less expressive logic than ours and not dealing with implementation issues.

We proceed as follows. The next section provides some background on mathematical fuzzy logics and (fuzzy) rough set theory. Section 3 presents the definition of a extension of the DL $\mathcal{S R O \mathcal { I }}(\mathbf{D})$, the logic behind OWL 2, with fuzzy and fuzzy rough semantics. Section 4 describes two reasoning algorithms under two fragments of our logic. Finally, Section 5 sets out some conclusions.

\section{Preliminaries}

Mathematical Fuzzy Logic. In fuzzy logics, the convention prescribing that a statement is either true or false is changed. Changing the usual true/false convention leads to a new concept of statement, whose compatibility with a given state of facts is a matter of degree and can be measured on an ordered scale $\mathcal{S}$ that is no longer $\{0,1\}$, but, e.g., the unit interval $[0,1]$. This degree of fit is called degree of truth of the statement $\phi$ in the interpretation $\mathcal{I}$.

Fuzzy logics logics provide compositional calculi of degrees of truth, including degrees between "true" and "false". A statement is now not true or false only, but may have a truth degree taken from a truth space $\mathcal{S}$, usually [0,1] (in that case we speak about Mathematical Fuzzy Logic [14]). In this paper, fuzzy statements will have the form $\phi \geqslant l$ or $\phi \leqslant u$, where $l, u \in[0,1]$ and $\phi$ is a statement, which encode that the degree of truth of $\phi$ is at least $l$ resp. at most $u$.

Semantically, a fuzzy interpretation $\mathcal{I}$ maps each basic statement $p_{i}$ into $[0,1]$ and is then extended inductively to all statements as follows:

$$
\begin{aligned}
& \mathcal{I}(\phi \wedge \psi)=\mathcal{I}(\phi) \otimes \mathcal{I}(\psi), \mathcal{I}(\phi \vee \psi)=\mathcal{I}(\phi) \oplus \mathcal{I}(\psi), \\
& \mathcal{I}(\phi \rightarrow \psi)=\mathcal{I}(\phi) \Rightarrow \mathcal{I}(\psi), \mathcal{I}(\neg \phi)=\ominus \mathcal{I}(\phi),
\end{aligned}
$$

where $\otimes, \oplus, \Rightarrow$, and $\ominus$ are so-called combination functions, namely, triangular norms (or t-norms), triangular conorms (or t-conorms), implication functions, and negation functions, respectively, which extend the classical Boolean conjunction, disjunction, implication, and negation, respectively, to the fuzzy case (see [14] for a formal definition of these functions and their properties). An important type of implication functions are $R$-implications, defined as $a \Rightarrow b=\sup \{c \mid a \otimes c \leq b\}$. 
Table 1. Combination functions of various fuzzy logics

\begin{tabular}{lcccc}
\hline & Lukasiewicz Logic & Gödel Logic & Product Logic & Zadeh Logic \\
\hline $\begin{array}{l}a \otimes b \\
a \oplus b\end{array}$ & $\begin{array}{l}\max (a+b-1,0) \\
\min (a+b, 1)\end{array}$ & $\min (a, b)$ & $\begin{array}{c}a \cdot b \\
\max (a, b)\end{array}$ & $\begin{array}{l}\min (a, b) \\
\max (a, b)\end{array}$ \\
$a \Rightarrow b$ & $\min (1-a+b, 1)$ & $\begin{cases}1 & \text { if } a \leq b \\
b & \text { otherwise }\end{cases}$ & $\min (1, b / a)$ & $\max (1-a, b)$ \\
$\ominus a$ & $1-a$ & $\begin{cases}1 & \text { if } a=0 \\
0 & \text { otherwise }\end{cases}$ & $\begin{cases}1 & \text { if } a=0 \\
0 & \text { otherwise }\end{cases}$ & $1-a$ \\
\hline
\end{tabular}

Several t-norms, t-conorms, implication functions, and negation functions have been proposed, giving raise to different fuzzy logics with different logical properties. In fuzzy logic, one usually distinguishes three different logics, namely Łukasiewicz, Gödel, and Product logic [14. Zadeh logic (the fuzzy operators originally considered by Zadeh [30]) is a sublogic of Eukasiewicz logic. Łukasiewicz, Gödel, and Product logics have an R-implication, while Zadeh logic does not.

A fuzzy set $R$ over a countable crisp set $X$ is a function $R: X \rightarrow[0,1]$. A fuzzy set $A$ is included in $B$ (denoted $A \subseteq B$ ) iff $\forall x \in X, A(x) \leq B(x)$. The degree of subsumption between two fuzzy sets $A$ and $B$ is defined as $\inf _{x \in X} A(x) \Rightarrow B(x)$.

A (binary) fuzzy relation $R$ over two countable crisp sets $X$ and $Y$ is a function $R: X \times Y \rightarrow[0,1]$. The inverse of $R$ is the function $R^{-1}: Y \times X \rightarrow[0,1]$ with membership function $R^{-1}(y, x)=R(x, y)$, for every $x \in X$ and $y \in Y$. The composition of two fuzzy relations $R_{1}: X \times Y \rightarrow[0,1]$ and $R_{2}: Y \times Z \rightarrow[0,1]$ is defined as $\left(R_{1} \circ R_{2}\right)(x, z)=\sup _{y \in Y} R_{1}(x, y) \otimes R_{2}(y, z)$. A fuzzy relation $R$ is reflexive iff $\forall x \in X, R(x, x)=1$. $R$ is symmetric iff $\forall x \in X, y \in Y, R(x, y)=$ $R(y, x) . R$ is transitive iff $R(x, z) \geqslant(R \circ R)(x, z)$. A fuzzy similarity relation is a reflexive, symmetric and transitive relation.

A fuzzy interpretation $\mathcal{I}$ satisfies a fuzzy statement $\phi \geqslant l$ (resp., $\phi \leqslant u$ ) or $\mathcal{I}$ is a model of $\phi \geqslant l$ (resp., $\phi \leqslant u$ ), denoted $\mathcal{I} \models \phi \geqslant l$ (resp., $\mathcal{I} \models \phi \leqslant u$ ), iff $\mathcal{I}(\phi) \geqslant l$ (resp., $\mathcal{I}(\phi) \leqslant u$ ). The notions of satisfiability and logical consequence are defined in the standard way. $\phi \geqslant l$ is a tight logical consequence of a set of fuzzy statements $\mathcal{K}$ iff $l$ is the infimum of $\mathcal{I}(\phi)$ subject to all models $\mathcal{I}$ of $\mathcal{K}$. Notice that the latter is equivalent to $l=\sup \{r \mid \mathcal{K} \models \phi \geqslant r\}$.

Rough Set and Fuzzy Rough Set Theories. The key idea in rough set theory [22] is the approximation of a vague concept by means of a pair a concepts: a sub-concept or lower approximation and a super-concept or upper approximation, describing the sets of elements which definitely and possibly belong to the vague set, respectively, as Figure 1 illustrates. The approximation is based on an indiscernibility equivalence relation (reflexive, symmetric and transitive) between elements of the domain. Given an indiscernibility relation $R$, the upper approximation of a set $S$ is defined as: $\bar{S}=\{x \mid \exists y:(x, y) \in R \wedge y \in S\}$. Similarly, the lower approximation is defined as: $\underline{S}=\{x \mid \forall y:(x, y) \in R \rightarrow y \in S\}$.

A very natural extension is to consider a fuzzy similarity relation instead of an indiscernibility relation, which gives raise to fuzzy rough sets 1123. Given a fuzzy similarity relation $R$, a t-norm $\otimes$ and an implication function $\Rightarrow$, the upper 


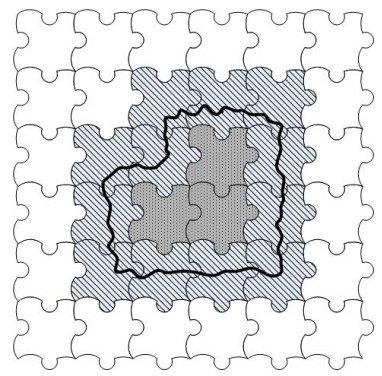

Fig. 1. Vague concept (bold line), upper approximation (striped line) and lower approximation (dotted line)

approximation of a fuzzy set $S$ is given by the following membership function: $\forall x \in X, \bar{S}(x)=\sup _{y \in \Delta^{\mathcal{I}}}\{R(x, y) \otimes S(y)\}$. Similarly, the lower approximation is defined as: $\forall x \in X, \underline{S}(x)=\inf _{y \in \Delta^{\mathcal{I}}}\{R(x, y) \Rightarrow S(y)\}$.

\section{The Fuzzy Rough DL $\mathcal{S} \mathcal{R} \mathcal{O} \mathcal{I}(\mathrm{D})$}

In this section we describe a fuzzy rough extension of the fuzzy DL $\mathcal{S} \mathcal{R O} \mathcal{I}(\mathbf{D})$, which is based on the fuzzy DLs presented in 5728 , and extended with upper and lower approximations of concepts. In the following, we assume $\bowtie \in\{\geq,>, \leq,<\}$, $\triangleright \in\{\geq,>\}, \triangleleft \in\{\leq,<\}, \alpha \in(0,1], \beta \in[0,1), \gamma \in[0,1]$.

Syntax. A fuzzy concrete domain 27] $\mathbf{D}$ is a pair $\left\langle\Delta_{\mathbf{D}}, \Phi_{\mathbf{D}}\right\rangle$, where $\Delta_{\mathbf{D}}$ is a concrete interpretation domain, and $\Phi_{\mathbf{D}}$ is a set of fuzzy concrete predicates $\mathbf{d}$ with an arity $n$ and an interpretation $\mathbf{d}_{\mathbf{D}}: \Delta_{\mathbf{D}}^{n} \rightarrow[0,1]$, which is an $n$-ary fuzzy relation over $\Delta_{\mathbf{D}}$. Usual functions for specifying fuzzy set membership degrees are the trapezoidal, the triangular, the $L$-function (left-shoulder function), and the $R$-function (right-shoulder function). For backwards compatibility, we also allow crisp intervals. These functions are defined over the set of non-negative rationals $\mathbb{Q}^{+} \cup\{0\}$ For instance, we may define Young: $\mathbb{N} \rightarrow[0,1]$ to be a fuzzy concrete predicate over the natural numbers denoting the degree of a person being young, as Young $(x)=L(10,30)$.

We further allow fuzzy modifiers, such as very, moreOrLess and slightly, which apply to fuzzy sets to change their membership function. Formally, a modifier is a function $f_{m}:[0,1] \rightarrow[0,1]$. We will allow modifiers defined in terms of linear hedges and triangular functions. For instance, very $(x)=\operatorname{linear}(0.8)$.

Similarly as for its crisp counterpart, fuzzy $\mathcal{S R O \mathcal { I }}(\mathbf{D})$ assumes three alphabets of symbols, for concepts, roles and individuals.

The abstract roles (denoted $R$ ) of the language can be built inductively as:

$$
\begin{array}{r|l}
R \rightarrow R_{A} & \mid \text { (atomic role) } \\
R^{-} & \text {(inverse role) } \\
U & \mid \text { (universal role) }
\end{array}
$$

Concrete roles are denoted $T$ and cannot be complex. 
Now, let $n, m$ be natural numbers $(n \geqslant 0, m>0)$. The concepts (denoted $C$ or $D$ ) of the language can be built inductively from atomic concepts $(A)$, top concept $\top$, bottom concept $\perp$, named individuals $\left(o_{i}\right)$, abstract roles $(R)$, concrete roles $(T)$, simple roles $(S) 1$ and fuzzy concrete predicates $(\mathbf{d})$ as:

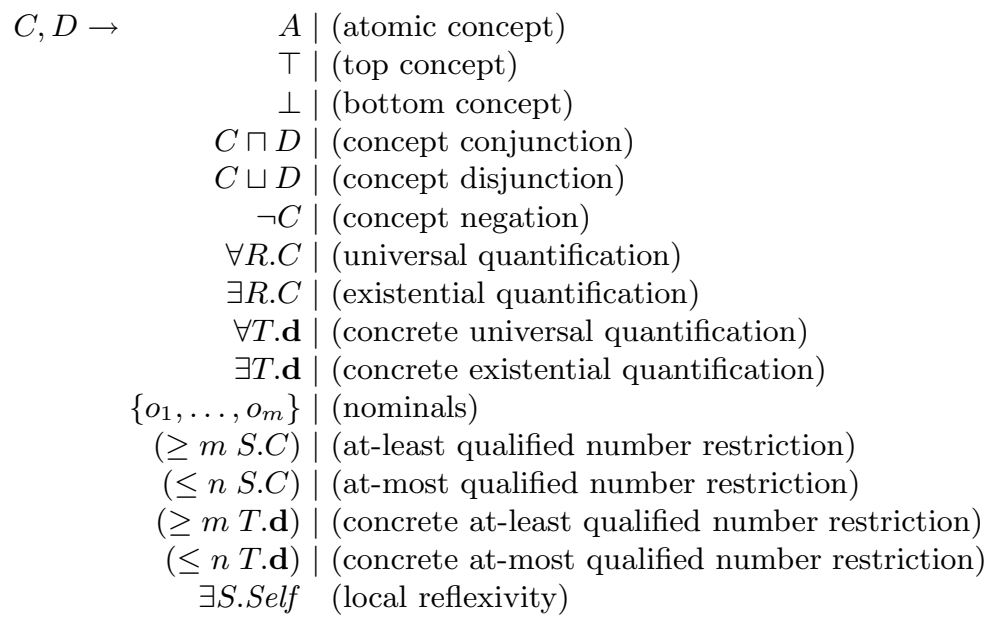

Assume $m$ fuzzy similarity relations $s_{i}(i=1, \ldots, m)$. The above syntax is extended to include salient features of fuzzy DLs 37 as follows:

$$
\begin{aligned}
& C, D \rightarrow\left\{\alpha_{1} / o_{1}, \ldots, \alpha_{m} / o_{m}\right\} \mid \text { (fuzzy nominals) } \\
& C \rightarrow D \mid \text { (fuzzy implication concept) } \\
& \alpha_{1} C_{1}+\cdots+\alpha_{k} C_{k} \mid \text { (fuzzy weighted sum) } \\
& \bmod (C) \mid(\text { modified concept) } \\
& {[C \geq \alpha] \mid \text { (cut concept) }} \\
& {[C \leq \beta] \mid \text { (cut concept) }} \\
& \bar{C}^{i} \mid \text { (upper approximation) } \\
& \underline{C}_{i} \text { (lower approximation) } \\
& \mathbf{d} \rightarrow \quad \operatorname{crisp}(a, b) \mid(\text { fuzzy crisp set) } \\
& L(a, b) \mid \text { (fuzzy left-shoulder function) } \\
& R(a, b) \mid \text { (fuzzy right-shoulder function) } \\
& \text { triangular }(a, b, c) \mid \text { (fuzzy triangular function) } \\
& \text { trapezoidal }(a, b, c, d) \quad \text { (fuzzy trapezoidal function) } \\
& \bmod \rightarrow \quad \text { linear }(c) \mid \text { (fuzzy linear modifier) } \\
& \text { triangular }(a, b, c) \text { (fuzzy triangular modifier) } \\
& R \rightarrow \quad \bmod (R) \mid(\text { modified role) } \\
& {[R \geq \alpha] \quad \text { (cut role) }}
\end{aligned}
$$

In the case of linear modifiers, we assume that $a=c /(c+1), b=1 /(c+1)$. Furthermore, for each of the connectives $\sqcap, \sqcup, \rightarrow$, we have indexed connectives

${ }^{1}$ Simple roles are needed to guarantee the decidability of the logic. Intuitively, simple roles cannot take part in cyclic role inclusion axioms (see [6] for a formal definition). 
$\sqcap_{X}, \sqcup_{X}, \rightarrow_{X}$, where $X \in\{$ Gödel, Łukasiewicz, Product\}, which are interpreted according to the semantics of the subscript.

Example 1. Concept Human $\sqcap \exists$ hasAge. $L(10,30)$ denotes the set of young humans, with an age given by $L(10,30)$. If linear(4) represents the modifier very, Human $\sqcap$ linear $(4)(\exists$ hasAge. $L(10,30))$ denotes the set of very young humans.

Furthermore, abstract individuals are denoted $a, b \in \Delta^{\mathcal{I}}$, while concrete individuals are denoted $v \in \Delta_{\mathbf{D}}$.

A Fuzzy Knowledge Base (KB) contains axioms organized in a fuzzy ABox $\mathcal{A}$, a fuzzy TBox $\mathcal{T}$ and a fuzzy RBox $\mathcal{R}$.

A fuzzy ABox consists of a finite set of fuzzy assertions of one of these types:

- a fuzzy concept assertion of the form $\langle a: C \bowtie \alpha\rangle$;

- a fuzzy role assertion, or constraint on the truth value of a role assertion, $\langle\Psi \bowtie \alpha\rangle$, where $\Psi$ is of the form $(a, b): R,(a, b): \neg R,(a, v): T$ or $(a, v): \neg T$;

- an inequality assertion $\langle a \neq b\rangle$;

- an equality assertion $\langle a=b\rangle$.

A fuzzy TBox consists of a finite set of fuzzy General Concept Inclusions or fuzzy $G C I s$, which are expressions of the form $\langle C \sqsubseteq D \geq \alpha\rangle$ or $\langle C \sqsubseteq D>\beta\rangle$.

A fuzzy $R B$ ox consists of a finite set of role axioms of one these types:

- Fuzzy Role Inclusion Axioms or fuzzy RIAs $\langle w \sqsubseteq R \geq \alpha\rangle,\langle w \sqsubseteq R>\beta\rangle$, where $w=R_{1} R_{2} \ldots R_{m}$ is a role chain, $\left\langle T_{1} \sqsubseteq T_{2} \geq \alpha\right\rangle$, or $\left\langle T_{1} \sqsubseteq T_{2}>\beta\right\rangle$;

- transitive role axioms $\operatorname{trans}(R)$;

- disjoint role axioms dis $\left(S_{1}, S_{2}\right)$, dis $\left(T_{1}, T_{2}\right)$;

- reflexive role axioms $\operatorname{ref}(R)$;

- irreflexive role axioms $\operatorname{irr}(S)$;

- symmetric role axiom $\operatorname{sym}(R)$;

- asymmetric role axioms asy $(S)$.

Example 2. 〈paul: Tall $\geq 0.5\rangle$ states that Paul is tall with at least degree 0.5. The fuzzy RIA 〈isFriendOf isFriendOf $\sqsubseteq$ isFriendOf $\geq 0.75$ > states that the friends of my friends can also be considered my friends with degree 0.75 .

A fuzzy axiom has a truth degree in $[0,1]$. A fuzzy axiom is positive (denoted $\langle\tau \triangleright \alpha\rangle)$ if it is of the form $\langle\tau \geq \alpha\rangle$ or $\langle\tau>\beta\rangle$. A fuzzy axiom is negative (denoted $\langle\tau \triangleleft \alpha\rangle)$ if it is of the form $\langle\tau \leq \beta\rangle$ or $\langle\tau<\alpha\rangle$.

Semantics. A fuzzy interpretation $\mathcal{I}$ with respect to a fuzzy concrete domain $\mathbf{D}$ is a pair $\left(\Delta^{\mathcal{I}},{ }^{\mathcal{I}}\right)$ consisting of a non empty set $\Delta^{\mathcal{I}}$ (the interpretation domain) disjoint with $\Delta_{\mathbf{D}}$ and a fuzzy interpretation function ${ }^{\mathcal{I}}$ mapping:

- an abstract individual a onto an element $a^{\mathcal{I}}$ of $\Delta^{\mathcal{I}}$;

- a concrete individual $v$ onto an element $v_{\mathbf{D}}$ of $\Delta_{\mathbf{D}}$;

- a concept $C$ onto a function $C^{\mathcal{I}}: \Delta^{\mathcal{I}} \rightarrow[0,1]$;

- an abstract role $R$ onto a function $R^{\mathcal{I}}: \Delta^{\mathcal{I}} \times \Delta^{\mathcal{I}} \rightarrow[0,1]$; 
- a concrete role $T$ onto a function $T^{\mathcal{I}}: \Delta^{\mathcal{I}} \times \Delta_{\mathbf{D}} \rightarrow[0,1]$;

- an $n$-ary concrete fuzzy predicate $\mathbf{d}$ onto the fuzzy relation $\mathbf{d}_{\mathbf{D}}: \Delta_{\mathbf{D}}^{n} \rightarrow[0,1]$;

- a modifier mod onto a function $f_{\text {mod }}:[0,1] \rightarrow[0,1]$.

Given arbitraries t-norm $\otimes$, t-conorm $\oplus$, negation function $\ominus$ and implication function $\Rightarrow$, the fuzzy interpretation function is extended to complex concepts and roles as shown in Table 2, and to fuzzy axioms as shown in Table 3.

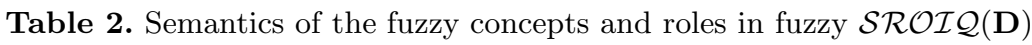

\begin{tabular}{|c|c|}
\hline Constructor & Semantics \\
\hline 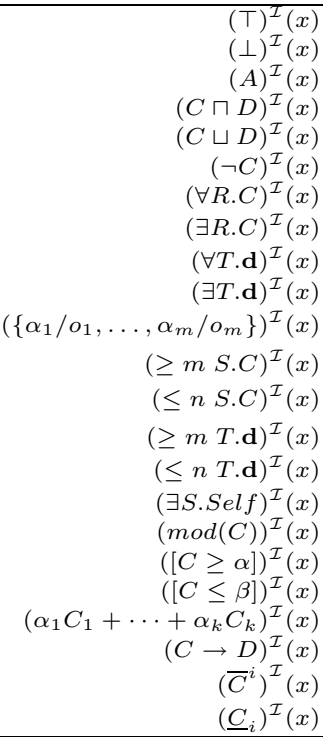 & 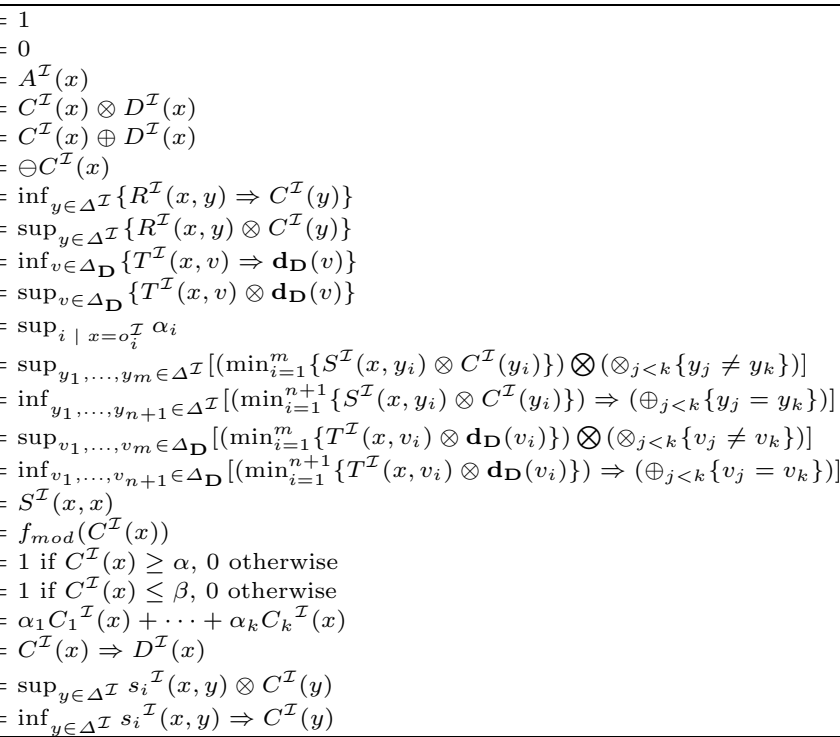 \\
\hline $\begin{array}{r}\left(R_{A}\right)^{\mathcal{I}}(x, y) \\
\left(R^{-}\right)^{\mathcal{I}}(x, y) \\
(U)^{\mathcal{I}}(x, y) \\
(\bmod (R))^{\mathcal{I}}(x, y) \\
([R \geq \alpha])^{\mathcal{I}}(x, y) \\
(T)^{\mathcal{I}}(x, v)\end{array}$ & $\begin{aligned} & =R_{A}^{\mathcal{I}}(x, y) \\
= & R^{\mathcal{I}}(y, x) \\
= & 1 \\
= & f_{\bmod }\left(R^{\mathcal{I}}(x, y)\right) \\
= & 1 \text { if } R^{\mathcal{I}}(x, y) \geq \alpha, 0 \text { otherwise } \\
= & T^{\mathcal{I}}(x, v)\end{aligned}$ \\
\hline
\end{tabular}

$C^{\mathcal{I}}$ denotes the membership function of the fuzzy concept $C$ with respect to the fuzzy interpretation $\mathcal{I}$. $C^{\mathcal{I}}(x)$ gives us the degree of being the individual $x$ an element of the fuzzy concept $C$ under $\mathcal{I}$.

Similarly, $R^{\mathcal{I}}$ denotes the membership function of the fuzzy role $R$ with respect to $\mathcal{I}$. $R^{\mathcal{I}}(x, y)$ gives us the degree of being $(x, y)$ an element of the fuzzy role $R$ under $\mathcal{I}$.

A fuzzy interpretation $\mathcal{I}$ satisfies (is a model of):

$-\langle a: C \bowtie \gamma\rangle$ iff $(a: C)^{\mathcal{I}} \bowtie \gamma$,

$-\langle(a, b): R \bowtie \gamma\rangle$ iff $((a, b): R)^{\mathcal{I}} \bowtie \gamma$,

$-\langle(a, b): \neg R \bowtie \gamma\rangle$ iff $((a, b): \neg R)^{\mathcal{I}} \bowtie \gamma$,

$-\langle(a, v): T \bowtie \gamma\rangle$ iff $((a, v): T)^{\mathcal{I}} \bowtie \gamma$, 
Table 3. Semantics of the fuzzy axioms in fuzzy $\mathcal{S} \mathcal{R O} \mathcal{I}(\mathbf{D})$

\begin{tabular}{|c|c|}
\hline Axiom & Semantics \\
\hline $\begin{aligned}(a: C)^{\mathcal{I}} \\
((a, b): R)^{\mathcal{I}} \\
\quad((a, b): \neg R)^{\mathcal{I}} \\
((a, v): T)^{\mathcal{I}} \\
((a, v): \neg T)^{\mathcal{I}} \\
(C \sqsubseteq D)^{\mathcal{I}} \\
\left(R_{1} \ldots R_{m} \sqsubseteq R\right)^{\mathcal{I}} \\
\left(T_{1} \sqsubseteq T_{2}\right)^{\mathcal{I}}\end{aligned}$ & 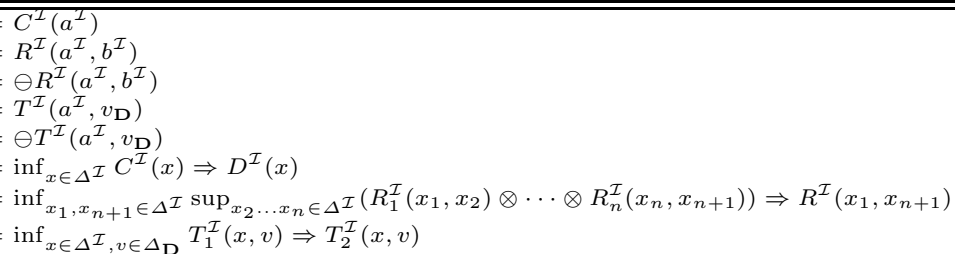 \\
\hline
\end{tabular}

$-\langle(a, v): \neg T \bowtie \gamma\rangle$ iff $((a, v): \neg T)^{\mathcal{I}} \bowtie \gamma$,

$-\langle a \neq b\rangle$ iff $a^{\mathcal{I}} \neq b^{\mathcal{I}}$,

$-\langle a=b\rangle$ iff $a^{\mathcal{I}}=b^{\mathcal{I}}$,

$-\langle C \sqsubseteq D \triangleright \gamma\rangle$ iff $(C \sqsubseteq D)^{\mathcal{I}} \triangleright \gamma$,

$-\left\langle R_{1} \ldots R_{m} \sqsubseteq R \triangleright \gamma\right\rangle$ iff $\left(R_{1} \ldots R_{m} \sqsubseteq R\right)^{\mathcal{I}} \triangleright \gamma$,

$-\left\langle T_{1} \sqsubseteq T_{2} \triangleright \bar{\gamma}\right\rangle$ iff $\left(T_{1} \sqsubseteq T_{2}\right)^{\mathcal{I}} \triangleright \gamma$,

- trans $(R)$ iff $\forall x, y \in \bar{\Delta}^{\mathcal{I}}, R^{\mathcal{I}}(x, y) \geq \sup _{z \in \Delta^{\mathcal{I}}} R^{\mathcal{I}}(x, z) \otimes R^{\mathcal{I}}(z, y)$,

$-\operatorname{dis}\left(S_{1}, S_{2}\right)$ iff $\forall x, y \in \Delta^{\mathcal{I}}, S_{1}^{\mathcal{I}}(x, y)=0$ or $S_{2}^{\mathcal{I}}(x, y)=0$,

- $\operatorname{dis}\left(T_{1}, T_{2}\right)$ iff $\forall x \in \Delta^{\mathcal{I}}, v \in \Delta_{\mathbf{D}}, T_{1}^{\mathcal{I}}(x, v)=0$ or $T_{2}^{\mathcal{I}}(x, v)=0$,

- $\operatorname{ref}(R)$ iff $\forall x \in \Delta^{\mathcal{I}}, R^{\mathcal{I}}(x, x)=1$,

$-\operatorname{irr}(S)$ iff $\forall x \in \Delta^{\mathcal{I}}, S^{\mathcal{I}}(x, x)=0$,

- $\operatorname{sym}(R)$ iff $\forall x, y \in \Delta^{\mathcal{I}}, R^{\mathcal{I}}(x, y)=R^{\mathcal{I}}(y, x)$,

- $\operatorname{asy}(S)$ iff $\forall x, y \in \Delta^{\mathcal{I}}$, if $S^{\mathcal{I}}(x, y)>0$ then $S^{\mathcal{I}}(y, x)=0$,

- a fuzzy KB iff it satisfies each element in $\mathcal{A}, \mathcal{T}$ and $\mathcal{R}$.

Reasoning. The notions of logical consequence and tight logical consequence are defined as in Sect. 2. Additionally, the maximal satisfiability degree 7] of a concept $C$ w.r.t. a fuzzy KB $\mathcal{K}$ is defined as $g l b(\mathcal{K}, C)=\sup _{\mathcal{I}} \sup _{x \in \Delta^{\mathcal{I}}} C^{\mathcal{I}}(x)$.

Some logical properties. Due to the properties of fuzzy rough sets 23, in Zadeh, Gödel, Łukasiewicz and Product logics we have that:

$-\bar{\perp} \equiv \underline{\perp} \equiv \perp, \underline{I} \equiv \bar{\top} \equiv \top, \underline{\underline{C}} \equiv \underline{C}, \overline{\bar{C}} \equiv \bar{C}$.

$-\neg \underline{C} \equiv \overline{\neg C}$, in Zadeh and Lukasiewicz logics.

$-\neg \bar{C} \equiv \neg C$, in Zadeh and Lukasiewicz logics.

$-\overline{C \sqcap D} \subseteq \bar{C} \sqcap \bar{D}, \underline{C \sqcap D} \equiv \underline{C} \sqcap \underline{D}$,

$-\overline{C \sqcup D} \equiv \bar{C} \sqcup \bar{D}$, in Zadeh and Gödel logics.

$-\underline{C \sqcup D} \supseteq \underline{C} \sqcup \underline{D}$.

Note that fuzzy rough intersection and union are not truth-functional in general.

\section{Reasoning and Implementation}

In this section we will show how to extend two existing reasoning algorithms for fuzzy DLs so they can support fuzzy rough DLs, and how we have implemented 
them in the FUzzYDL system [7] and in the DeLorean system [4]. To this end, we recall that indeed we can map lower and upper approximation concepts into fuzzy DL concepts. This is not surprising as already pointed out by 24] for the crisp case. In fact, it is not difficult to see from the semantics of upper $\left(\bar{C}^{i}\right)$ and lower $\left(\underline{C}_{i}\right)$ approximation concepts, that these can be represented as fuzzy DL concepts $\exists s_{i} . C$ and $\forall s_{i} . C$, respectively. That is, we consider the transformation:

$$
\begin{aligned}
& \bar{C}^{i} \mapsto \exists s_{i} . C \\
& \underline{C}_{i} \mapsto \forall s_{i} . C
\end{aligned}
$$

and, thus, we may replace upper and lover approximation concepts with ordinary fuzzy DL concepts. This is exactly the same transformation as provided in 24. In the following, we show how two currently highly expressive fuzzy DL reasoners have been adapted to support our logic.

\subsection{Tableau Rules and an Optimization Problem in fuzzyDL}

FUZZYDL is a reasoner for fuzzy $\mathcal{S H \mathcal { I }} \mathcal{F}(\mathbf{D})$ extended with a lot of salient features of fuzzy DLs, under Zadeh, Łukasiewicz and Gödel logics [7. It is available from http://www.straccia.info, and supports the logic defined in Sect. 3 without the additional elements of $\mathcal{S} \mathcal{R O I} \mathcal{Q}$, i.e., fuzzy nominals, qualified cardinality restrictions, role assertions with a negated role, disjoint role axioms, complex fuzzy RIAs (with $w \neq R$ ), irreflexive role axioms and asymmetric role axioms.

Its reasoning algorithm combines a tableaux algorithm and a mixed integer linear optimization problem. The basic idea is to build a tableaux using a set of satisfiability preserving rules which generate new simpler fuzzy assertion axioms together with some inequations over [0,1]-valued variables. Finally, an optimization problem through the set of inequations is solved. A detailed description of the reasoning algorithm cannot fit into this paper, but it can be found in 29].

To support upper and lower approximation concepts in FUZZYDL, essentially we need to support reflexive roles (symmetric and transitive roles are already supported). In particular, we firstly extend FUZZYDL with a couple of fuzzy role axioms. Reflexive and symmetric role axioms are of the form (reflexive R) and (symmetric R), respectively, where R is a fuzzy role. Symmetric role axioms can already be simulated with FUZZYDL, and this axioms is just syntactic sugar. Indeed, axiom $R \sqsubseteq R^{-}$implies that $R$ is symmetric.

Then, we allow three additional concept constructors: upper approximations, lower approximations and local reflexivity concepts, which are of the form (ua $s_{i}$ C), ( $\operatorname{la~}_{\mathrm{i}} \mathrm{C}$ ) and ( $\mathrm{self} \mathrm{S}$ ), respectively, where $\mathrm{s}_{\mathrm{i}}$ is a fuzzy similarity relation, $\mathrm{S}$ is a simple fuzzy role and $\mathrm{C}$ is a fuzzy concept. Local reflexivity concepts are not necessary for the rough extension, but adding them is easy (reasoning is similar to the case of reflexive roles).

Similarity relations must be previously defined using the following syntax: (define-fuzzy-similarity $\mathrm{s}_{\mathrm{i}}$ ). 
The reasoning algorithm is extended as follows:

- For every fuzzy similarity relation (define-fuzzy-similarity $s_{i}$ ) we assert $\mathrm{s}_{\mathrm{i}}$ to be reflexive, symmetric and transitive by adding the following axioms: (reflexive R), (symmetric R), (transitive R).

- Every symmetric role axiom (symmetric R) is replaced with an inverse role axiom (inverse $R$ invR) and a role inclusion axiom (implies-role $R$ invR). Under an R-implication, it is well known that sym( $R)$ is equivalent to $\mathrm{R} \sqsubseteq \mathrm{R}^{-}$.

- Every upper approximation concept (ua $s_{i} C$ ) is replaced with an existential restriction concept (some $s_{i} C$ ).

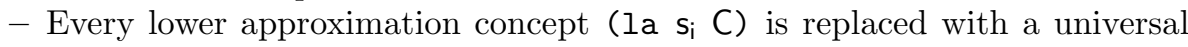
restriction concept (all $\mathrm{s}_{\mathrm{i}} \mathrm{C}$ ).

- The rule for a local reflexivity concept (self S) asserts that an individual is related to itself. Formally, in the calculus if $\langle\exists \mathrm{S}$.Self, $l\rangle \in \mathcal{L}(v)$ (that is, if $v$ is an instance of $\exists$ S.Self to degree not smaller than $l$ ) then append $\langle\mathrm{S}, l\rangle$ to $\mathcal{L}(\langle v, v\rangle$ ) (that is, the pair $\langle v, v\rangle$ is an instance of $\mathrm{S}$ at least to degree $l$ ).

- The rule for reflexive roles (reflexive $R$ ) asserts that every individual is related to itself. Formally, if $\langle\operatorname{ref}(\mathrm{R})\rangle \in \mathcal{R}$ and $v$ is a node to which this rule has not yet been applied then append $\langle\mathrm{R}, l\rangle$ to $\mathcal{L}(\langle v, v\rangle$ ) (that is, the pair $\langle v, v\rangle$ is an instance of $\mathrm{R}$ to degree not smaller than $l$ ).

\subsection{Reduction to Classical Description Logic in DeLorean}

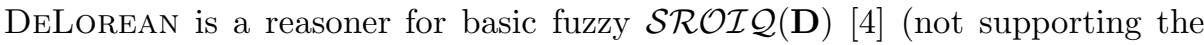
additional features of fuzzy DLs defined in Sect. 3) under Zadeh and Gödel (with an involutive negation) logics. The syntax of the supported language is in $[2]$.

Its reasoning algorithm is based on a reduction to a classical DL, so current DL reasoners can be reused. A full description may be found in 356 .

DELOREAN already supported local reflexivity concepts, as well as reflexive and symmetric roles. Hence, it only remained to extend it with upper and lower approximations of the form (upper $s_{i} C$ ) and (lower $s_{i} C$ ), where $s_{i}$ is a fuzzy similarity relation and $C$ is a fuzzy concept.

Now, the reasoning algorithm is extended as follows:

- Every concept (upper $s_{i} C$ ) is replaced with an existential restriction concept (some $s_{i}$ C). Furthermore, we add the following axioms if they do not exist in the fuzzy RBox: (reflexive R), (symmetric R), (transitive R).

- Every concept (lower $s_{i} C$ ) is replaced with a universal restriction concept (all $\mathrm{s}_{\mathrm{i}} \mathrm{C}$ ). Once again, we add the following axioms in case they do not exist in the fuzzy RBox: (reflexive R), (symmetric R), (transitive R).

\section{Conclusions}

In this paper we have studied a DL managing vagueness in two different but complementary ways, combining a fuzzy DL with fuzzy rough sets. In particular, we 
have presented a very expressive fuzzy rough extension of the DL $\mathcal{S} \mathcal{R O} \mathcal{I}(\mathbf{D})$, the logic behind the language OWL 2. The rough extension is general (independent of the family of fuzzy operators) and uses $m$ possible fuzzy similarity relations.

Reasoning under our general fuzzy rough DL is not currently possible, but we have extended and implemented two well-known reasoning algorithms for fuzzy DLs in order to deal with two important fragments of the logic. On the one hand, FUZZYDL implements a combination of a tableaux algorithm and a mixed integer linear optimization problem, and already supports fuzzy rough $\mathcal{S H \mathcal { F }} \mathcal{F}(\mathbf{D})$ (extended with salient features of fuzzy DLs) under Zadeh, Łukasiewicz and Gödel logics. On the other hand, DELOREAN implements a translation to a crisp DL

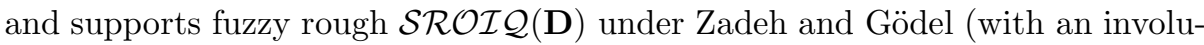
tive negation) logics. Extending the of reasoning algorithms and the expressivity reasoners remains an open research problem.

\section{References}

1. Baader, F., Calvanese, D., McGuinness, D., Nardi, D., Patel-Schneider, P.F. (eds.): The Description Logic Handbook: Theory, Implementation, and Applications. Cambridge University Press, Cambridge (2003)

2. Bobillo, F.: Managing Vagueness in Ontologies. PhD thesis, University of Granada, Spain (2008)

3. Bobillo, F., Delgado, M., Gómez-Romero, J.: A crisp representation for fuzzy $\mathcal{S H O I N}$ with fuzzy nominals and general concept inclusions. In: Uncertainty Reasoning for the Semantic Web I. LNCS, vol. 5327, pp. 174-188. Springer, Heidelberg (2008)

4. Bobillo, F., Delgado, M., Gómez-Romero, J.: DeLorean: A reasoner for fuzzy OWL 1.1. In: Proc. of the 4th International Workshop on Uncertainty Reasoning for the Semantic Web (URSW 2008). CEUR Workshop Proceedings, vol. 423 (2008)

5. Bobillo, F., Delgado, M., Gómez-Romero, J.: Optimizing the crisp representation of the fuzzy description logic $\mathcal{S} \mathcal{R O} \mathcal{I} \mathcal{Q}$. In: Uncertainty Reasoning for the Semantic Web I. LNCS, vol. 5327, pp. 189-206. Springer, Heidelberg (2008)

6. Bobillo, F., Delgado, M., Gómez-Romero, J., Straccia, U.: Fuzzy description logics under Gödel semantics. International Journal of Approximate Reasoning 50(3), 494-514 (2009)

7. Bobillo, F., Straccia, U.: Fuzzydl: An expressive fuzzy description logic reasoner. In: Proceedings of the 17th IEEE International Conference on Fuzzy Systems (FUZZIEEE 2008), pp. 923-930. IEEE Computer Society, Los Alamitos (2008)

8. Cuenca-Grau, B., Horrocks, I., Motik, B., Parsia, B., Patel-Schneider, P., Sattler, U.: OWL 2: The next step for OWL. Journal of Web Semantics 6(4), 309-322 (2008)

9. Dey, L., Abulaish, M., Goyal, R., Shubham, K.: A rough-fuzzy ontology generation framework and its application to bio-medical text processing. In: Proceedings of the 5th Atlantic Web Intelligence Conference (AWIC 2007), pp. 74-79 (2007)

10. Doherty, P., Grabowski, M., Łukaszewicz, W., Szałas, A.: Towards a framework for approximate ontologies. Fundamenta Informaticae 57(2-4), 147-165 (2003)

11. Dubois, D., Prade, H.: Rough fuzzy sets and fuzzy rough sets. International Journal of General Systems 17(2-3), 191-209 (1990) 
12. Fanizzi, N., D'Amato, C., Esposito, F., Lukasiewicz, T.: Representing uncertain concepts in rough description logics via contextual indiscernibility relations. In: Proceedings of the 4th International Workshop on Uncertainty Reasoning for the Semantic Web (URSW 2008). CEUR Workshop Proceedings, vol. 423 (2008)

13. Gruber, T.R.: A translation approach to portable ontologies. Knowledge Acquisition 5(2), 199-220 (1993)

14. Hájek, P.: Metamathematics of Fuzzy Logic. Kluwer Academic Publishers, Dordrecht (1998)

15. Hájek, P.: Making fuzzy description logics more general. Fuzzy Sets and Systems 154(1), 1-15 (2005)

16. Horrocks, I., Patel-Schneider, P.: Reducing OWL entailment to description logic satisfiability. Journal of Web Semantics 1(4), 345-357 (2004)

17. Jiang, Y., Wang, J., Peng, D., Tang, S.: Reasoning within expressive fuzzy rough description logics. Fuzzy sets and systems (to appear)

18. Jiang, Y., Wang, J., Tang, S., Xiao, B.: Reasoning with rough description logics: An approximate concepts approach. Information Sciences 179(5), 600-612 (2009)

19. Klein, M.C.A., Mika, P., Schlobach, S.: Rough description logics for modeling uncertainty in instance unification. In: Proceedings of URSW 2007. CEUR Workshop Proceedings, vol. 327 (2007)

20. Liau, C.-J.: On rough terminological logics. In: Proc. of the 4th Int. Workshop on Rough Sets, Fuzzy Sets and Machine Discovery (RSFD 1996), pp. 47-54 (1996)

21. Lukasiewicz, T., Straccia, U.: Managing uncertainty and vagueness in description logics for the semantic web. Journal of Web Semantics 6(4), 291-308 (2008)

22. Pawlak, Z.: Rough sets. International Journal of Computer and Information Sciences 11, 341-356 (1982)

23. Radzikowska, A.M., Kerre, E.E.: A comparative study of fuzzy rough sets. Fuzzy Sets and Systems 126(2), 137-155 (2002)

24. Schlobach, S., Klein, M.C.A., Peelen, L.: Description logics with approximate definitions - Precise modeling of vague concepts. In: Proceedings of the 20th International Joint Conference on Artificial Intelligence (IJCAI 2007), pp. 557-562 (2007)

25. Stoilos, G., Stamou, G., Pan, J.Z., Tzouvaras, V., Horrocks, I.: Reasoning with very expressive fuzzy description logics. Journal of Artificial Intelligence Research 30(8), 273-320 (2007)

26. Straccia, U.: Reasoning within fuzzy description logics. Journal of Artificial Intelligence Research 14, 137-166 (2001)

27. Straccia, U.: Description logics with fuzzy concrete domains. In: Proceedings of the 21st Conference on Uncertainty in Artificial Intelligence (UAI 2005) (2005)

28. Straccia, U.: A fuzzy description logic for the semantic web. In: Sanchez, E. (ed.) Fuzzy Logic and the Semantic Web. Capturing Intelligence, pp. 73-90. Elsevier, Amsterdam (2006)

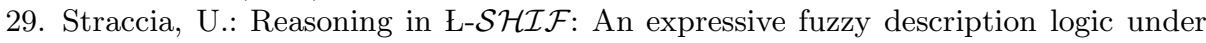
Łukasiewicz semantics. Technical Report TR-2007-10-18, Istituto di Scienza e Tecnologie dell'Informazione, Consiglio Nazionale delle Ricerche, Pisa, Italy (2007)

30. Zadeh, L.A.: Fuzzy sets. Information and Control 8(3), 338-353 (1965) 